\title{
Divergent interaction profiles of gabapentin and levetiracetam with dipalmitoylphosphatidylcholine lipids
}

\author{
Sevgi Türker-Kaya*, Aygül Kına, Selen Alın \\ Kocaeli University, Faculty of Arts and Sciences, Department of Biology, 41380, Kocaeli, Turkey
}

\section{A R T I C L E I N F O}

\section{Article history:}

Received 31 January 2017

Received in revised form 11 July 2017

Accepted 13 September 2017

Available online 14 September 2017

\section{Keywords:}

Gabapentin

Levetiracetam

Dipalmitoylphosphatidylcholines

Fourier transform infrared spectroscopy

Differential scanning calorimetry

\begin{abstract}
A B S T R A C T
Background/Objectives: The lipid solubility of antiepileptic drugs directly affects central nervous system availability. In relation to this, the interactions of gabapentin and levetiracetam with dipalmitoylphosphatidylcholine lipids depending on concentrations were comparatively investigated in the present study.

Methods: The effects of gabapentin and levetricetam as a function of concentration (1-5-10-20 mol\%) on biophysical parameters of dipalmitoylphosphatidylcholine multilammelar vesicles were studied by differential scanning calorimetry and fourier transform infrared spectroscopy.

Results: The data revealed that gabapentin at all concentrations and levetiracetam at 1-5 mol\% lowered main transition temperature, enthalpy, cooperativity, lipid fluidity, lipid order, and increased hydrogen binding capacity of glycerol and phosphate groups. However, 10-20 mol\% of levetiracetam tend to show different effect on transition temperature, which could also reflect its opposing effect on lipid order and glycerol and phosphate group's hydrations.

Conclusions: According to the corresponding findings depending on concentrations both drugs incorporate into phosphatidylcholines, perturbing the packing of lipids and affecting their thermotropic properties. Their binding affinity to acyl chains and hydrophilic parts of lipids was found to highly correlate with lipid-water partition and their solubility degree in water. Hence, the obtained results may offer evaluation of partition profile of the drugs into biological membranes depending on concentration. (C) 2017 Indian Epilepsy Society. Published by Elsevier, a division of RELX India, Pvt. Ltd. All rights reserved.
\end{abstract}

\section{Introduction}

Voltage-dependent sodium and calcium channel blockers and $\gamma$-aminobutyric acid (GABA) mimetics are approved as the most clinically useful groups of antiepileptic drugs (AEDs) to control the persistence of epileptic seizures. ${ }^{1}$ Gabapentin (GBP) (Fig. 1A), marketed under brand name Neurontin, is one of the drugs belonging to this group. It is a structural analogue of the inhibitory neurotransmitter GABA. ${ }^{2}$ Even though its action mechanism has not been fully clarified, ${ }^{3}$ GBP has been shown to increase GABA levels in brain ${ }^{4}$ and non-synaptic GABA neurotransmission. ${ }^{5}$ In addition; it has potency to reduce voltage-dependent calcium ${ }^{6}$ and sodium currents. ${ }^{7}$ Another drug called levetiracetam (LEV) (Fig. 1B), trade name Keppra, is also with unresolved mechanism of action to suppress epileptic seizures. ${ }^{8}$ However, evidence have

\footnotetext{
* Correspondence to: Department of Biology, Faculty of Arts and Sciences, 41380, Kocaeli,Turkey.

E-mail addresses: sevgitrkr@gmail.com, sevgi.turker@kocaeli.edu.tr (S. Türker-Kaya).
}

shown that unlike traditional AEDs, the antiepileptic effect of LEV is based on inhibition of hypersynchronization of epileptiform burst firing, therefore producing an inhibition of the spread of seizure activity. ${ }^{9}$ It is also considered to interact with a synaptic vesicle glycoprotein ${ }^{10}$ and inhibit presynaptic calcium channels, hence reducing neurotransmitter release. ${ }^{11}$ Additionally, its variable effects on synaptic transmission at hippocampal synapses have been reported. ${ }^{11}$ Depending on clinical studies, both GBP and LEV are accepted as safe and well-tolerated with promising pharmacokinetic properties. ${ }^{2,8}$

Like many other drug molecules, for absorption into the bloodstream and distribution throughout the body both GBP and LEV must cross one or more phospholipid bilayers to reach their site of action and to elicit their response in the brain. Even if their targets are not the lipid bilayer but rather cytosolic proteins or bilayer-inserted proteins, bilayer insertion is prerequisite to acquire their correct orientations upon contacting with lipid bilayer and diffuse along these bilayers to meet their targets. ${ }^{12}$ Thus, molecular interactions of such drugs with membrane lipids determine their orientation and conformation in membrane systems, and hence play an important role in transport, 


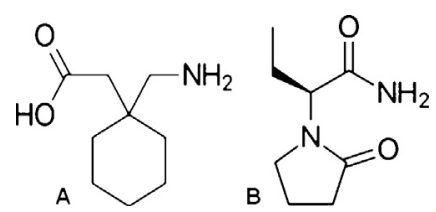

Fig. 1. Chemical structure of GBP (A) and LEV (B).

distribution, accumulation, and eventually their efficacy. ${ }^{13,14}$ More importantly, they affect their central nervous system availability. Therefore, the affinity of these drugs to membrane can provide useful information for the understanding of their partition profile into biological membranes, and relatively effectiveness. This may further develop potent drugs for epilepsy treatment as suggested by others. ${ }^{13}$

Considering the solubility in water and low protein binding capacity of GBP and LEV they have higher propensity to interact with membrane lipids. This may also facilitate their rapid and ready crossing of blood brain barrier. ${ }^{15,16}$ However; there is no published study yet available reporting how GBP and LEV partition into membrane lipids. On the other hand, since cell membrane consists of many different types of lipids, it is difficult to interpret interactions of drug with certain types of lipids with studies using whole cell membrane fraction. ${ }^{17}$ Moreover, experiments using whole cells or natural cell membrane patches are often time and cost-intensive, and there is lack of suitable techniques for real time measurement. The use of artificial membrane systems is a general approach for such research. Relatively, with aiming to provide a general perspective about how GBP and LEV incorporate into membrane lipids their interactions with dipalmitoylphosphatidylcholine (DPPC) multilamellar vesicles (MLVs) were comparatively investigated in the present study; as earlier works performed for different kinds of molecules. ${ }^{18-20}$ DPPC phospholipid bilayers used in the current work are considered suitable model systems to mimic cell membranes, and thus to study membrane structure and properties since phosphatidylcholines (PCs) are major lipid components of eukaryotic cell membrane. Additionally, their structural and thermodynamic properties are well-defined, which may be needed for the research of drug-lipid interactions. ${ }^{18,20}$ Thus, the interactions of GBP and LEV with DPPC MLVs in terms of lipid phase behavior, order and dynamics and hydration state of the polar part of DPPC MLVs were examined using differential scanning calorimetry (DSC) and Fourier transform infrared (FT-IR) spectroscopy, which have not been previously reported to the best of our knowledge.

\section{Material and methods}

\subsection{Chemicals}

Gabapentin [1-(Aminomethyl) cyclohexaneacetic acid], levetiracetam [(S)-2-(2-Oxopyrrolidin-1-yl)butanamide], dipalmitoylphosphatidylcholine (DPPC) and phosphate buffered saline (PBS) tablets were purchased from Sigma (St. Louis, MO, USA). All chemicals were obtained from commercial sources at the highest grade of purity available.

\subsection{Liposome preparation}

By following the previous reports ${ }^{18,20}$ DPPC MLVs in the absence and presence of GBP and LEV (1-5-10-20 mol\%) were prepared. Briefly, $5 \mathrm{mg}$ DPPC lipids were dissolved in chloroform and evaporated under nitrogen flow and desiccated under vacuum overnight to remove solvent. Then, thin films of dried lipids were obtained and re-suspended in phosphate buffer, pH 7.4. MLVs were formed by vigorous vortexing the mixture for $30 \mathrm{~min}$ at least $15^{\circ} \mathrm{C}$ above main phase transition temperature of DPPC lipids. In order to produce drug containing MLVs, the required amount from stock solution was initially placed in tube. The excess of organic solvent was removed by a stream of nitrogen, DPPC in chloroform was added and MLVs was prepared as described above.

\subsection{FT-IR studies and analysis}

$20 \mu \mathrm{L}$ of liposomes with and without drugs, and buffer placed between calcium florur $\left(\mathrm{CaF}_{2}\right)$ windows with $12 \mu \mathrm{m}$ sample thickness were scanned using a Perkin Elmer Spectrum 100 FT-IR spectrometer (Perkin Elmer, Inc., Norwalk, CT, USA) equipped with a deuterated triglycine sulfate (DTGS) detector. The experimentation were performed at $25-60^{\circ} \mathrm{C}$ to investigate if the drugs behave differently in the gel and the liquid crystalline phase of DPPC MLVs. Before the scans the samples were incubated for $5 \mathrm{~min}$. The interferograms were averaged for 100 scans at $2 \mathrm{~cm}^{-1}$ resolution. All experiments were three times performed. The sample compartment in the FT-IR spectrometer was continuously purged with dry air to prevent water vapor. The spectrum of air was automatically subtracted by Perkin Elmer Spectrum One software.

To improve resolution of the infrared bands of interest, buffer spectrum at corresponding temperature was subtracted to eliminate overlapping effect of the $\mathrm{OH}$ stretching modes (3400$3200 \mathrm{~cm}^{-1}$ and $1800-1500 \mathrm{~cm}^{-1}$ ) from water molecules in buffer. The subtraction process was performed till bulk water region located around $2100 \mathrm{~cm}^{-1}$ was flattened using same program.

Perkin Elmer Spectrum One software was used to determine variations in peak positions and bandwidths, as well. The band positions were measured from the center of weight $(0.80 \times$ peak height positions) and bandwidth values were calculated as the width at $0.75 \times$ height of the signal in terms of $\mathrm{cm}^{-1}$. For visual demonstration of the spectral differences in the spectra, the spectra were normalized with respect to the specific bands. Data analysis was performed on water subtracted sample spectra.

\subsection{DSC studies}

For DSC studies, MLVs were prepared by following the procedure in FT-IR sample preparation mentioned above. $50 \mu \mathrm{L}$ MLVs suspensions were encapsulated in hermetically sealed standard aluminum DSC pans. An indium containing pan was used as reference during the analysis. Measurements were performed using a Shimadzu DSC-60 Calorimeter (Shimadzu Corporation, Tokyo, Japan) equipped ascending and descending temperature mode operations. Scans were made at $0.5^{\circ} \mathrm{C} / \mathrm{min}$. Only heating curves are presented. Samples were scanned three times to ensure the reproducibility of the endotherms and to eliminate the thermal history of the sample. Data were analyzed using TA 60 software provided by Shimadzu. The temperature at the peak maximum was defined as the transition temperature. The enthalpy $\left(\Delta \mathrm{H}_{\text {cal }}\right)$ values were calculated by integrating the peak area under main transition. Cooperativity unit (CU), which is a measure of the mean number of lipid molecules undergoing transitions, was calculated as in Turker et al.. ${ }^{18}$

\subsection{Statistical analysis}

The mean of at least three experiments was plotted and calculated together with the standard error of mean in the figures and tables. Statistical significance was assessed using MannWhitney nonparametric test. Significant differences were statistically considered at the level of $\mathrm{p} \leq 0.05$. 


\section{Results}

In the present study, the effects of different concentrations of GBP and LEV on hydration state, lipid dynamics (fluidity), lipid acyl chain flexibility (ordering) and phase behavior properties of DPPC MLVs were examined by DSC and FT-IR spectroscopy. Results from extensive studies of drug-lipid interactions have been already published, primarily using DSC, where thermally induced phase transitions were investigated. Moreover, to draw solid conclusions and to provide thorough understanding and interpretation of calorimetric data, FT-IR spectroscopy was also included within the scope of the study.

In DSC studies, two endothermic transitions were exhibited in 25-60 ${ }^{\circ} \mathrm{C}$ range for DPPC MLVs (Fig. 2 and 3). The pre-transition peak $(T \mathrm{p})$ corresponds to the conversion of lamellar gel phase to rippled gel phase, and represents low enthalpy transition attributed to the mobility of choline and polar head groups of DPPC. The main transition peak $(T \mathrm{~m})$, reflects the transition from gel phase to lamellar liquid-crystalline phase belonging to the mobility of the alkyl chains. In the present study, Tm value of pure DPPC MLVs was obtained as $41.18 \circ \mathrm{C}$, which is consistent with the literature. ${ }^{18,19}$ Fig. 2 and 3 represent that the presence of GBP and LEV (1-5-10-20 mol\%) caused abolishment of pre-transition peak. Table illustrates the values of $T \mathrm{~m}, \Delta \mathrm{H}_{\mathrm{cal}}$ and CU of DPPC MLVs in the absence and presence of GBP and LEV with different concentrations. As shown in the table, incorporation of both GBP and LEV was found to alter the main phase transition with cooperativity of lipids. Particularly, 1-5-10-20 mol\% of GBP and $1-5 \mathrm{~mol} \%$ of LEV lowered $\mathrm{Tm}, \Delta \mathrm{H}_{\mathrm{cal}}$ and cooperativity. However, this trend was not obtained above $10-20 \mathrm{~mol} \%$ concentration of LEV. Indeed, LEV at concentrations of $10-20 \mathrm{~mol} \%$ led to an increase in Tm while still reducing $\Delta \mathrm{H}_{\text {cal }}$ and cooperativity of DPPC MLVs.

For FT-IR spectral interpretation, to conform to DSC analysis and the effects of GBP and LEV on frequency values of the $\mathrm{CH}_{2}$ asymmetric $\left(2925 \mathrm{~cm}^{-1}\right)$, the $\mathrm{C}=\mathrm{O}\left(1735 \mathrm{~cm}^{-1}\right)$ and the $\mathrm{PO}^{-}{ }_{2}$ $\left(1222 \mathrm{~cm}^{-1}\right)$, symmetric stretching modes were measured in the range of $25-60^{\circ} \mathrm{C}$. Additionally, the bandwidth values of the $\mathrm{CH}_{2}$ asymmetric stretching values were calculated. Fig. 4 shows representative FT-IR spectra of DPPC MLVs in the absence and presence of GBP and LEV at $20 \mathrm{~mol} \%$ in the liquid crystalline phase $\left(50^{\circ} \mathrm{C}\right)$, in the region of $3050-2800 \mathrm{~cm}^{-1}$. As represented in the figure, the $\mathrm{CH}_{2}$ asymmetric stretching mode was sufficiently separated after water subtraction, therefore; it was not necessary band deconvolution or fitting procedures to evaluate relative

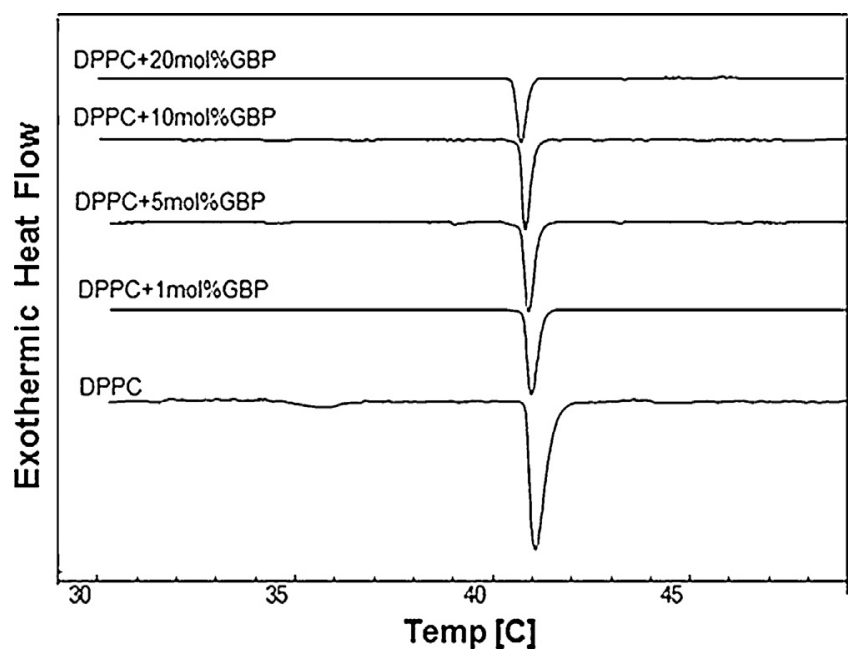

Fig. 2. Thermograms of DPPC liposomes in the absence and presence of different concentrations of GBP.

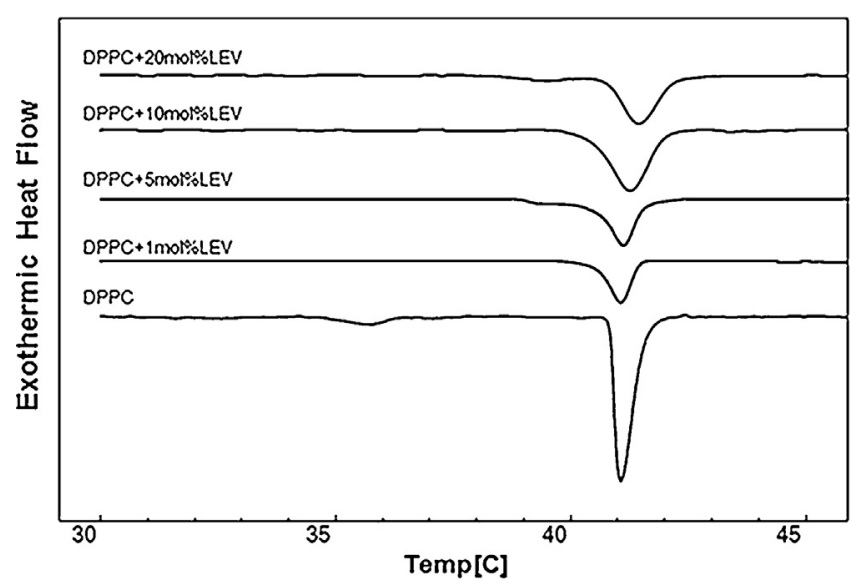

Fig. 3. Thermograms of DPPC liposomes in the absence and presence of different concentrations of LEV.

measurements for this study, as also suggested by the others. ${ }^{18,20}$ The spectra were normalized with respect to the $\mathrm{CH}_{2}$ asymmetric stretching mode to visually demonstrate the comparative difference in the frequency and bandwidth values of bands in the region of interest. The changes in order-disorder state (e.g. acyl chain flexibility) of DPPC MLVs due to the presence of the drugs were studied by monitoring of the $\mathrm{CH}_{2}$ asymmetric stretching mode frequency. The wavenumber values below $\mathrm{Tm}$ for DPPC is characteristic of conformationally ordered acyl chains with a high content of trans isomers, whereas the degrees at temperatures above $\mathrm{Tm}$ is of conformationally disordered acyl chains with a high content of gauche conformers. ${ }^{18,21}$ As shown in Fig. 5, GBP caused shifting of the frequency values of this band to higher degrees in both gel and liquid crystalline phases at all concentrations. The similar behavior was also observed by the addition of $1-5 \mathrm{~mol} \%$ of LEV but not for 10-20 mol\% (Fig. 6). The effects of GBP and LEV on dynamics (fluidity) of DPPC MLVs were studied by measuring the bandwidth values of the $\mathrm{CH}_{2}$ asymmetric stretching mode. ${ }^{20}$ Table 1 also demonstrates the changes in the bandwidth values of all samples at two different temperatures corresponding to gel and liquid crystalline phases of DPPC MLVs. According to the table, GBP at all concentrations (1-5-10-20 mol\%) and LEV at $1-5 \mathrm{~mol} \%$ decreased but LEV at $10-20$ mol\% slightly increased bandwidth values of the mode.

The frequency values of the $\mathrm{C}=\mathrm{O}$ mode monitor the hydration state of the glycerol backbone near the head group of phospholipids in interfacial region of the bilayer. ${ }^{18}, 20$ The temperature dependent frequency variations of this mode for GBP and LEV are shown in Fig. 7 and 8, respectively. The other mode called $\mathrm{PO}^{-}{ }_{2}$ symmetric stretching gives information about head groups of phospholipids. ${ }^{18}, 22$ Fig. 9 and 10 depict the changes in the frequency values of $\mathrm{PO}^{-}{ }_{2}$ asymmetric vibration band of DPPC with the absence and the presence of GBP and LEV. With the addition of GBP (1-5-10-20 mol\%) and LEV (1-5 mol\%) was found to result in a decrease but $10-20 \mathrm{~mol} \%$ of LEV was found to increase for in the frequencies of the $\mathrm{C}=\mathrm{O}$ and $\mathrm{PO}^{-}{ }_{2}$ modes. A decrement may indicate higher hydrogen bonding capacity of glycerol backbone and head groups of DPPC lipids. ${ }^{20}, 22$

\section{Discussion}

Both GBP and LEV are expected to interact with membrane lipids and partition into membrane due to having low molecular weight and not binding to plasma proteins. In particular, the partition coefficient values, measured octanol-water system, $\log \mathrm{P}$ at $\mathrm{pH} 7.4$, which is -1.10 and -0.6 for GBP ${ }^{23}$ and LEV ${ }^{24}$ 


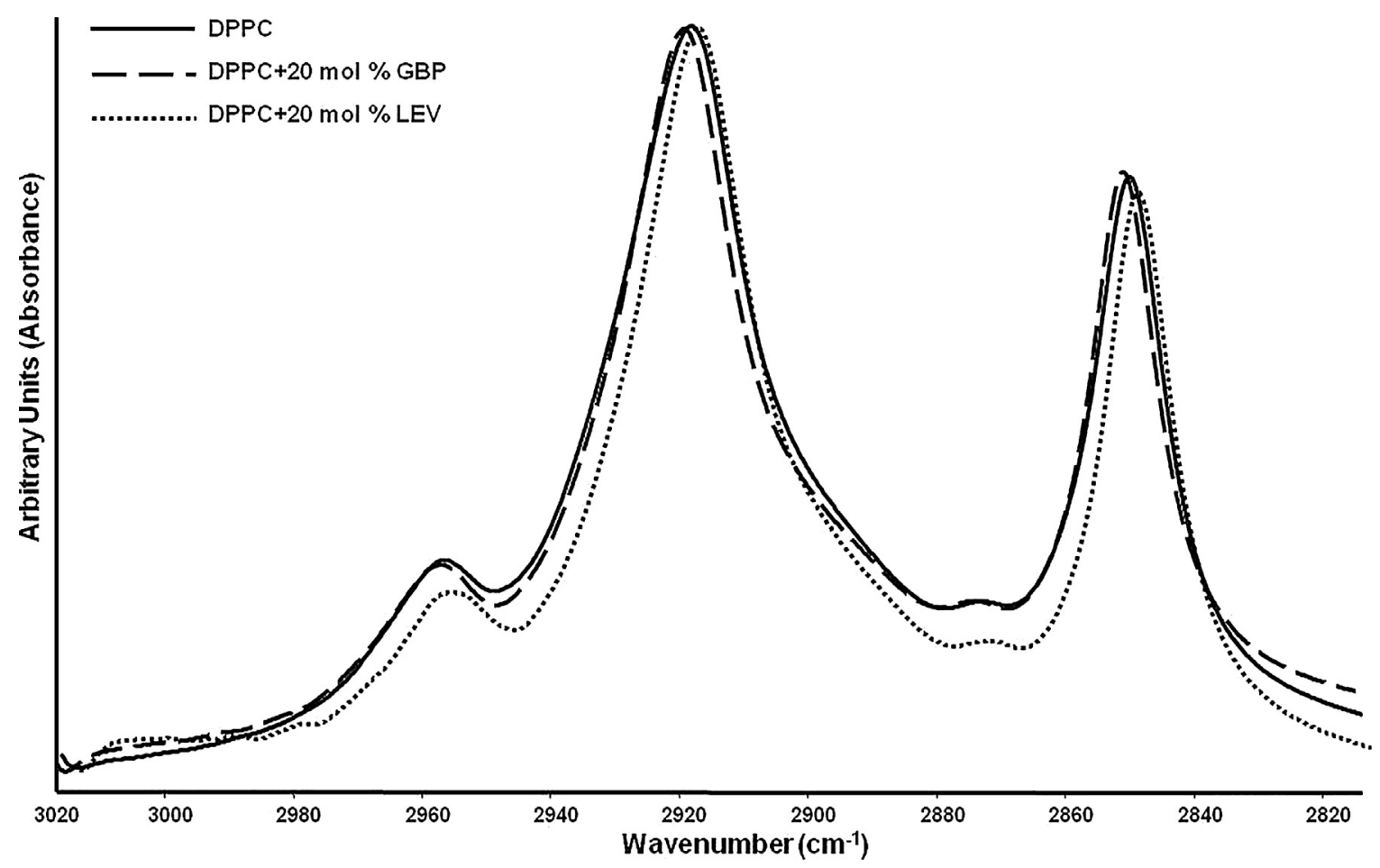

Fig. 4. Infrared spectra of DPPC liposomes in the absence and the presence of GBP and LEV at $50^{\circ} \mathrm{C}$ in the $3050-2800 \mathrm{~cm}^{-1}$ region. (The spectra were normalized with respect to the $\mathrm{CH}_{2}$ asymmetric stretching mode at $2925 \mathrm{~cm}^{-1}$ ).

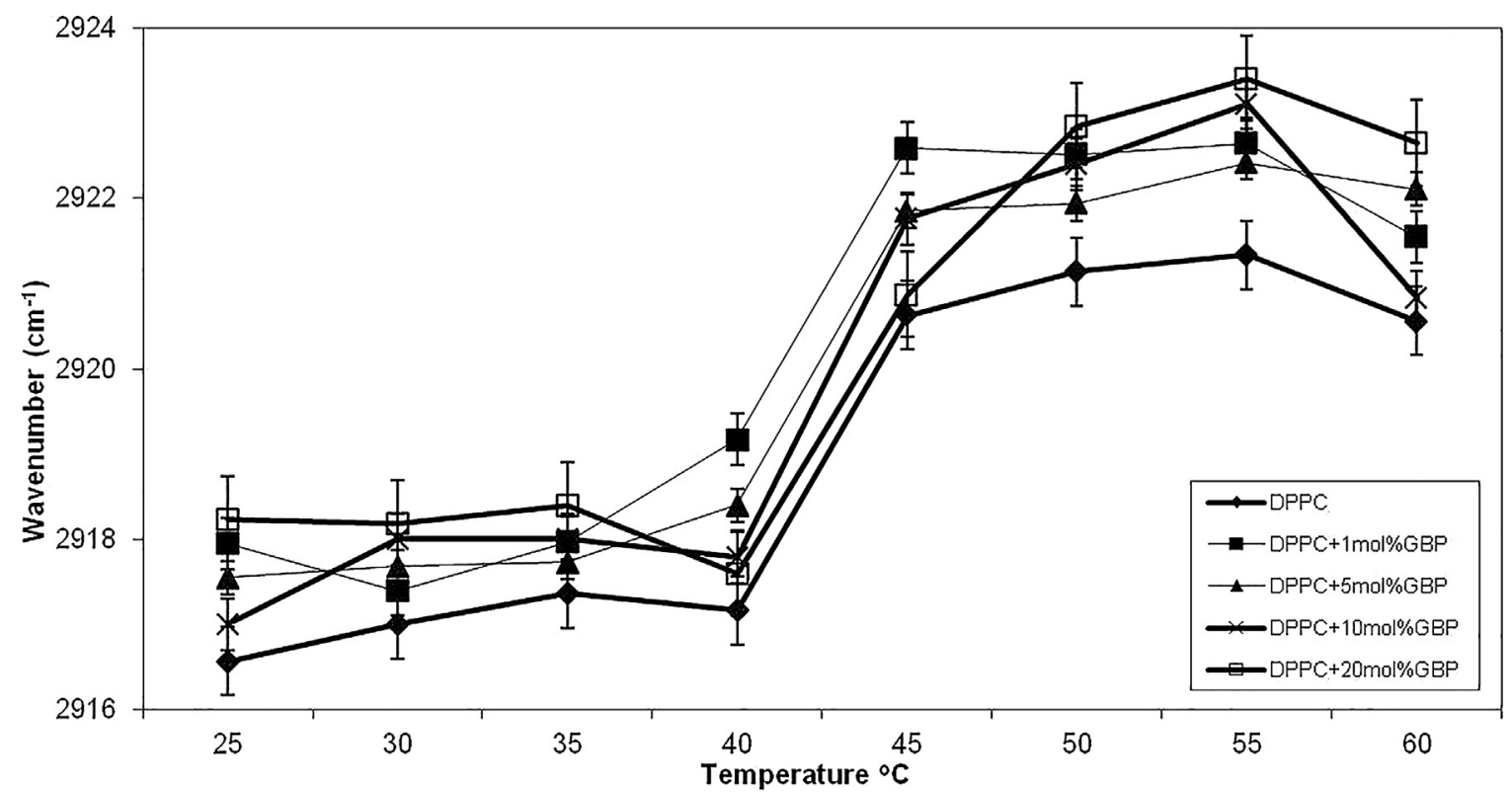

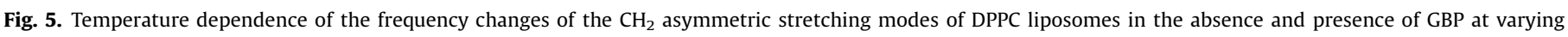
concentrations.

respectively, assume their lipophilic character. However, it should be mentioned that octanol-water system is free of ester and amide groups present in phospholipids, therefore; it may not represent behavior of the drugs of interest in natural membrane systems. For that reason, with aiming to contribute to clarifying the interaction potency of GBP and LEV with membrane lipids was evaluated in the present study. To achieve this, the interactions of these drugs with DPPC MLVs were examined by DSC and FT-IR spectroscopy, both of which have been widely employed for these kinds of studies. DPPC lipids used in the current study show spontaneous self-assembly into bilayers and readily form multilamellar with definite interlayer distance when dispersed in water, which render biological membranes their basic lamellar structure. Thus, they stand out to mimic cell membrane. ${ }^{20}$

According to DSC results, the pre-transition peak is abolished upon addition of both GBP and LEV in fully hydrated DPPC lipid bilayer, revealing perturbation of ripple phase. This may be a consequence of an extreme broadening that it is not reflected in heat capacity. ${ }^{25}$ In addition to that explanation, when ripple formation is coupled to size mismatch created by PCs head group, 


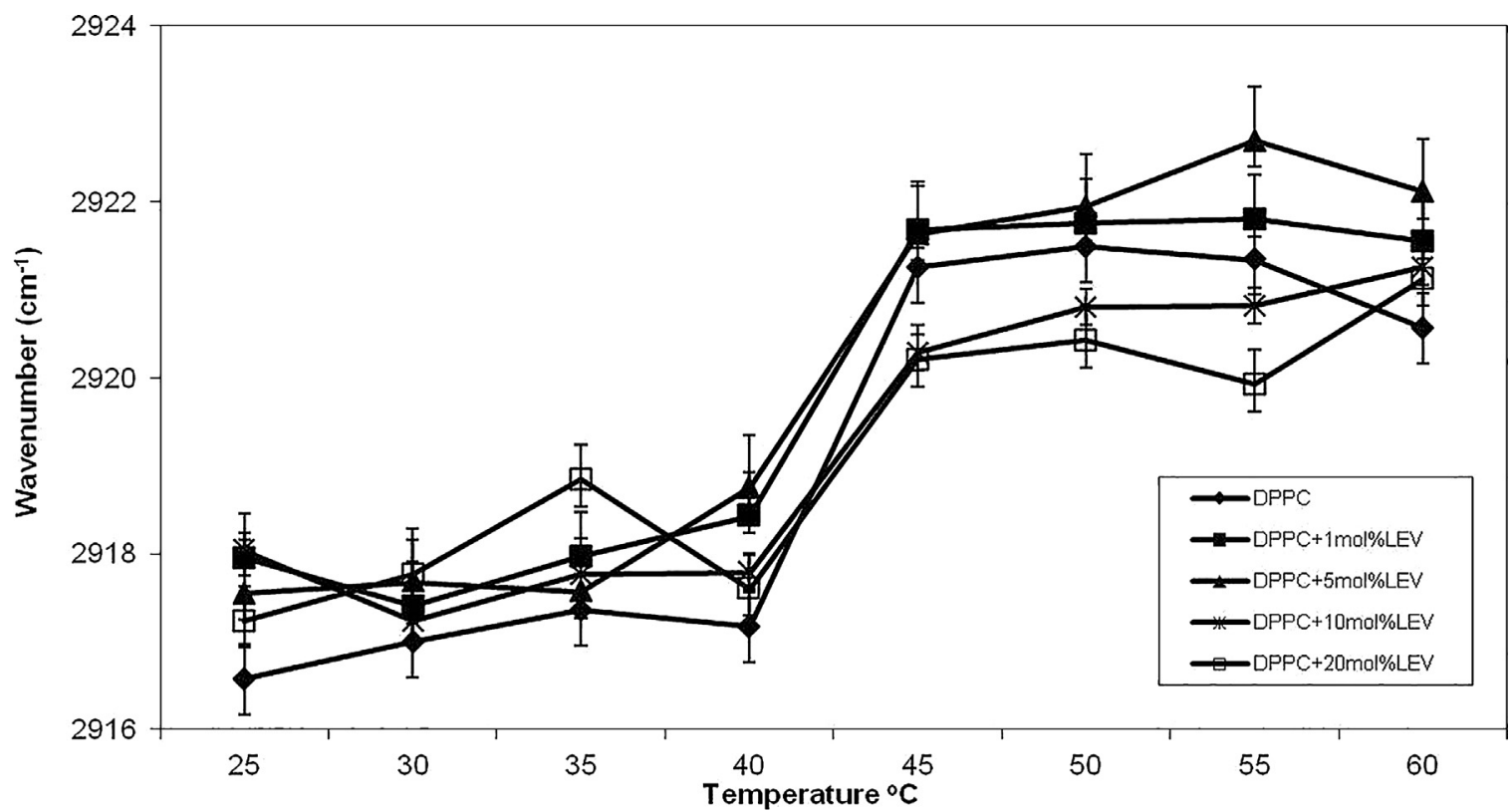

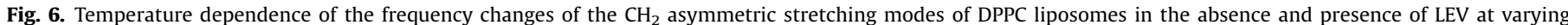
concentrations.

Table 1

$\mathrm{Tm}$, enthalpy, $\mathrm{CU}$ and $\mathrm{CH}_{2}$ asymmetric stretching bandwidth values of DPPC liposomes in the absence and presence of GBP and LEV.

\begin{tabular}{|c|c|c|c|c|}
\hline Sample & $\mathrm{Tm}$ & $\Delta$ Hcal & $\mathrm{CU}$ & $\mathrm{CH}_{2}$ asymmetric stretching bandwidth \\
\hline DPPC & $41.18 \pm 0.70$ & $43.04 \pm 0.09$ & $110.47 \pm 2.02$ & $26.05 \pm 1.09$ \\
\hline $\mathrm{DPPC}+1 \mathrm{~mol} \% \mathrm{GBP}$ & $41.09 \pm 0.22$ & $24.88 \pm 0.15^{* *}$ & $90.21 \pm 1.98^{*}$ & $25.15 \pm 1.07$ \\
\hline DPPC + 5 mol\%GBP & $41.06 \pm 0.16$ & $23.70 \pm 0.71^{* *}$ & $90.07 \pm 2.13^{*}$ & $25.05 \pm 0.74$ \\
\hline $\mathrm{DPPC}+10 \mathrm{~mol} \% \mathrm{GBP}$ & $40.99 \pm 0.55^{*}$ & $23.68 \pm 0.51^{* *}$ & $89.46 \pm 2.36^{*}$ & $25.08 \pm 1.88$ \\
\hline $\mathrm{DPPC}+20 \mathrm{~mol} \% \mathrm{GBP}$ & $40.87 \pm 0.09^{*}$ & $23.41 \pm 0.04^{* *}$ & $89.60 \pm 1.01^{*}$ & $24.00 \pm 0.13^{*}$ \\
\hline DPPC + 1 mol\%LEV & $41.12 \pm 0.33$ & $31.87 \pm 0.94^{* *}$ & $91.66 \pm 1.68^{*}$ & $25.88 \pm 0.04$ \\
\hline DPPC + 5 mol\%LEV & $40.85 \pm 0.08^{*}$ & $30.89 \pm 0.47^{* *}$ & $90.46 \pm 3.08^{*}$ & $25.87 \pm 1.76$ \\
\hline $\mathrm{DPPC}+10$ mol\%GBP & $40.99 \pm 0.11$ & $30.75 \pm 0.55^{* *}$ & $91.33 \pm 1.77^{*}$ & $25.11 \pm 0.09$ \\
\hline $\mathrm{DPPC}+20 \mathrm{~mol} \% \mathrm{GBP}$ & $41.14 \pm 0.17$ & $31.58 \pm 0.88^{* *}$ & $92.44 \pm 1.66^{*}$ & $24.19 \pm 0.03^{*}$ \\
\hline
\end{tabular}

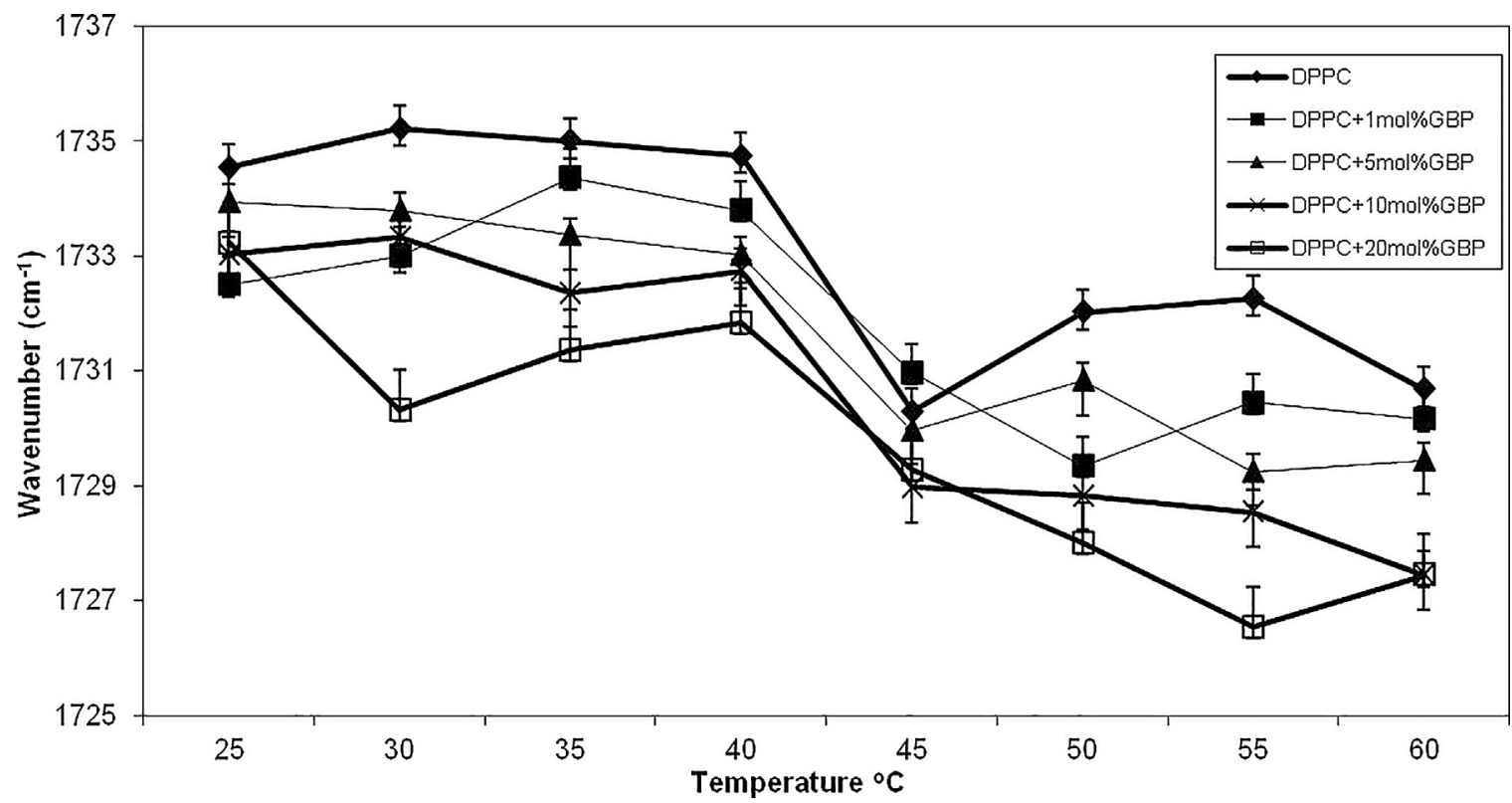

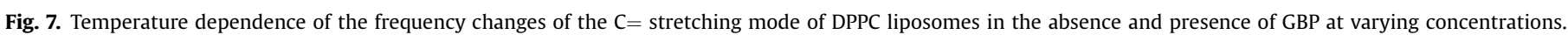




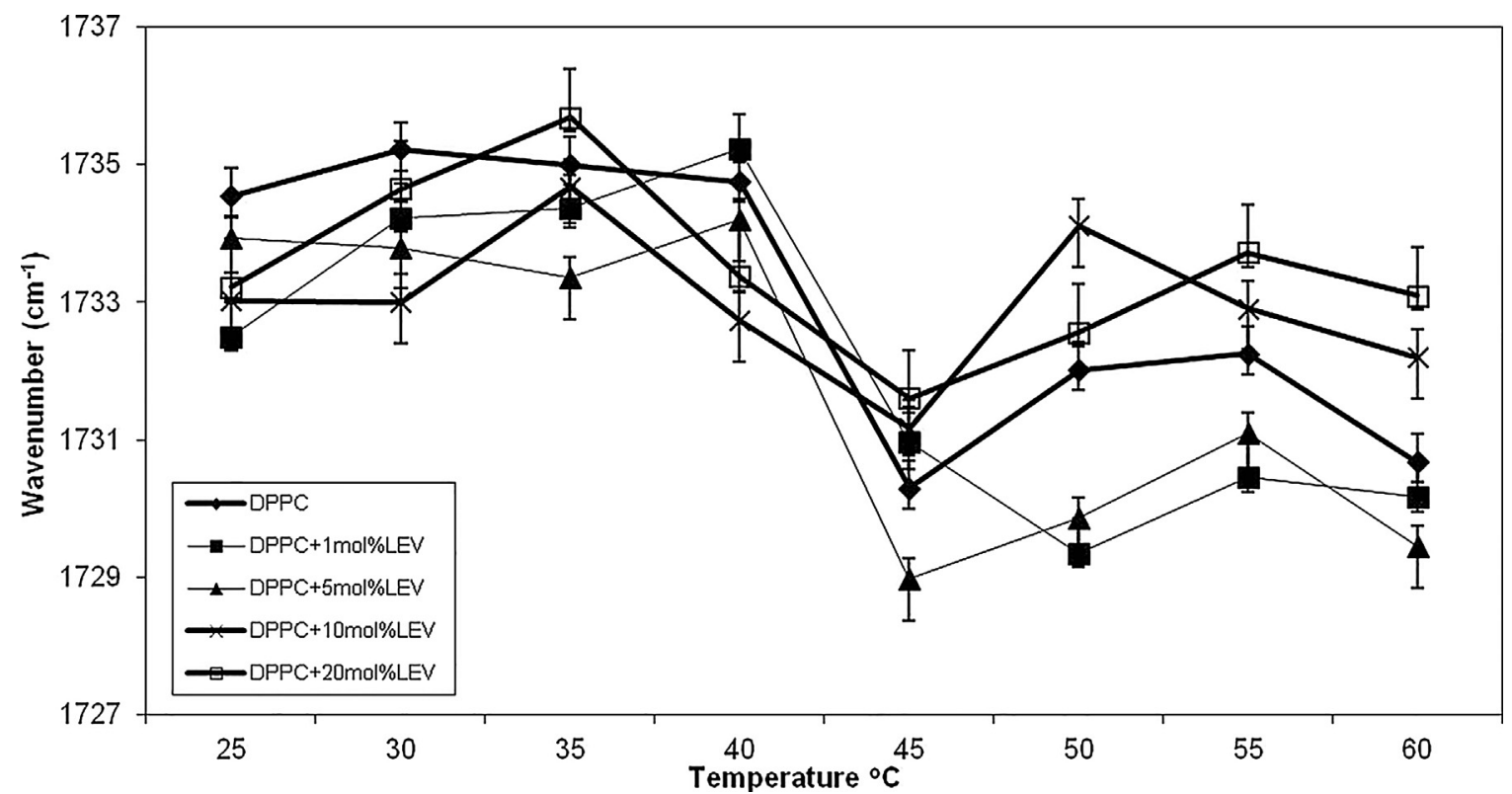

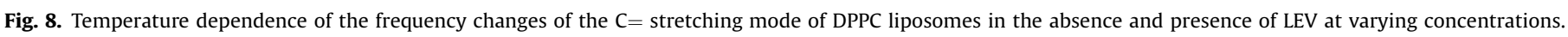

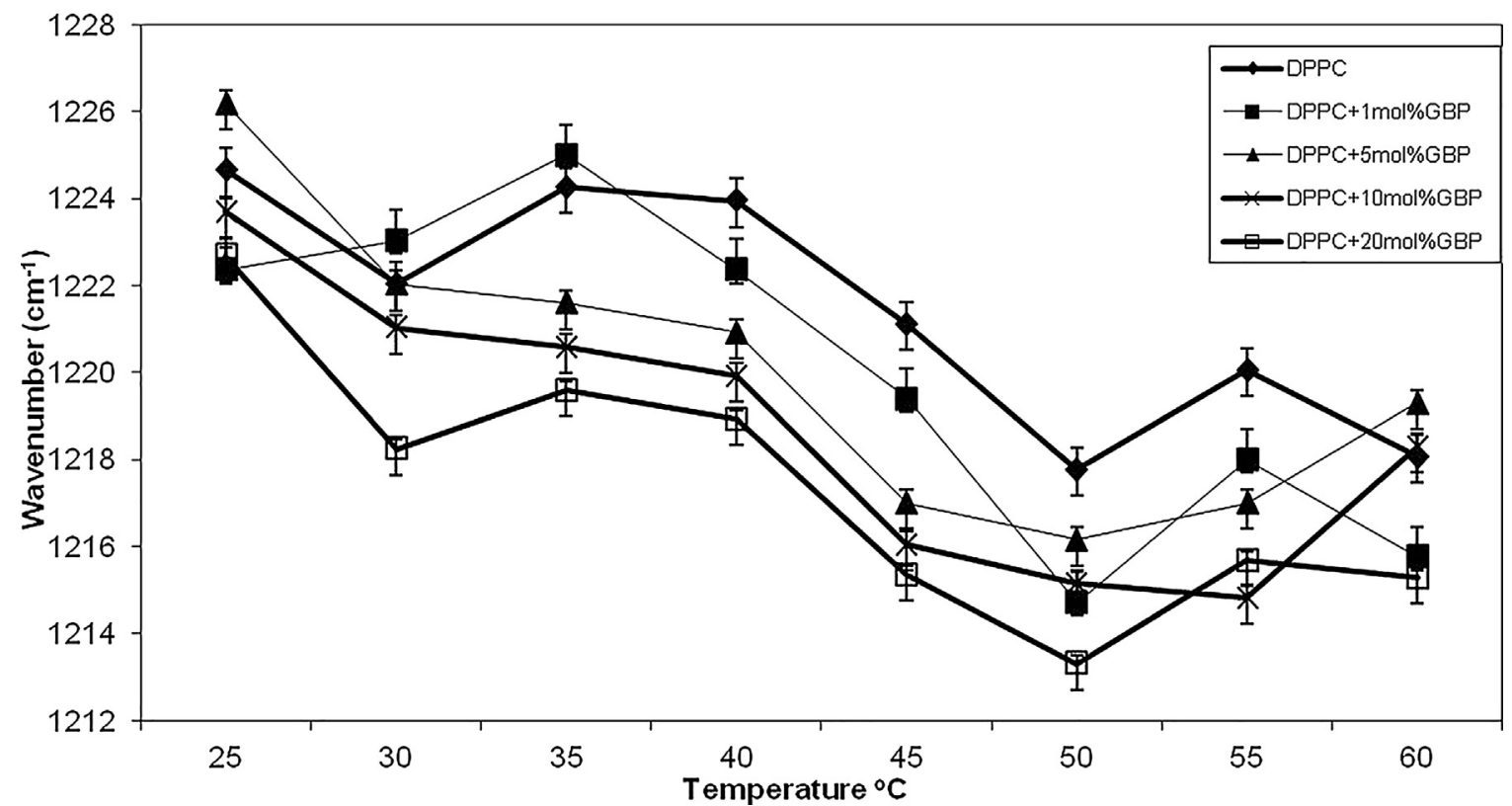

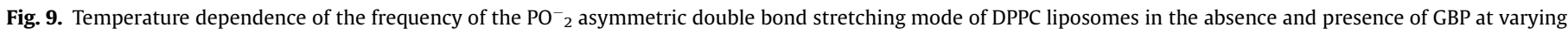
concentrations.

head group hydration plays crucial role in pre-transition. In other words, pre-transition of lipids is linked to periodic ripples on the membrane surface. ${ }^{26}$ Taking account of this, disappearance of pretransition induced by GBP and LEV may suggest that there is influence of these drugs at the surface of phospholipid vesicles, which may be resulted from any interaction. Similar result has been also reported for pentylenetetrazole ${ }^{18}$; and some anesthetics. ${ }^{27}$ However, it is still worthwhile stating that as partially soluble molecules in water GBP and LEV may accumulate at the surface of the bilayer. Since pre-transition is sensitive to other molecules in polar region of phospholipids, the disappearance of pre-transition peak cannot be accepted as a marker of interaction with hydrophilic part of lipids. ${ }^{18}$
The molecular interactions that control the main phase behavior may be dominated by lipid-lipid contacts and binding of any molecules. For that reason, any change in main transition can be referred partition of foreign molecule into lipids. In the current study, the main transition of DPPC MLVs was found to be broad in the presence of GBP and LEV. This can be also demonstrated from lower degree of $C U$ which indicates a decrease in the number of molecules that undergo transition simultaneously. 28 Our results showed that incorporation of GBP at all concentrations and LEV at concentrations of $1-5 \mathrm{~mol} \%$ into DPPC MLVs caused a reduction in Tm, enthalpy and CU, leading to reduced peak height. The lowered enthalpy together with broadening of transition represent that GBP and LEV perturb 


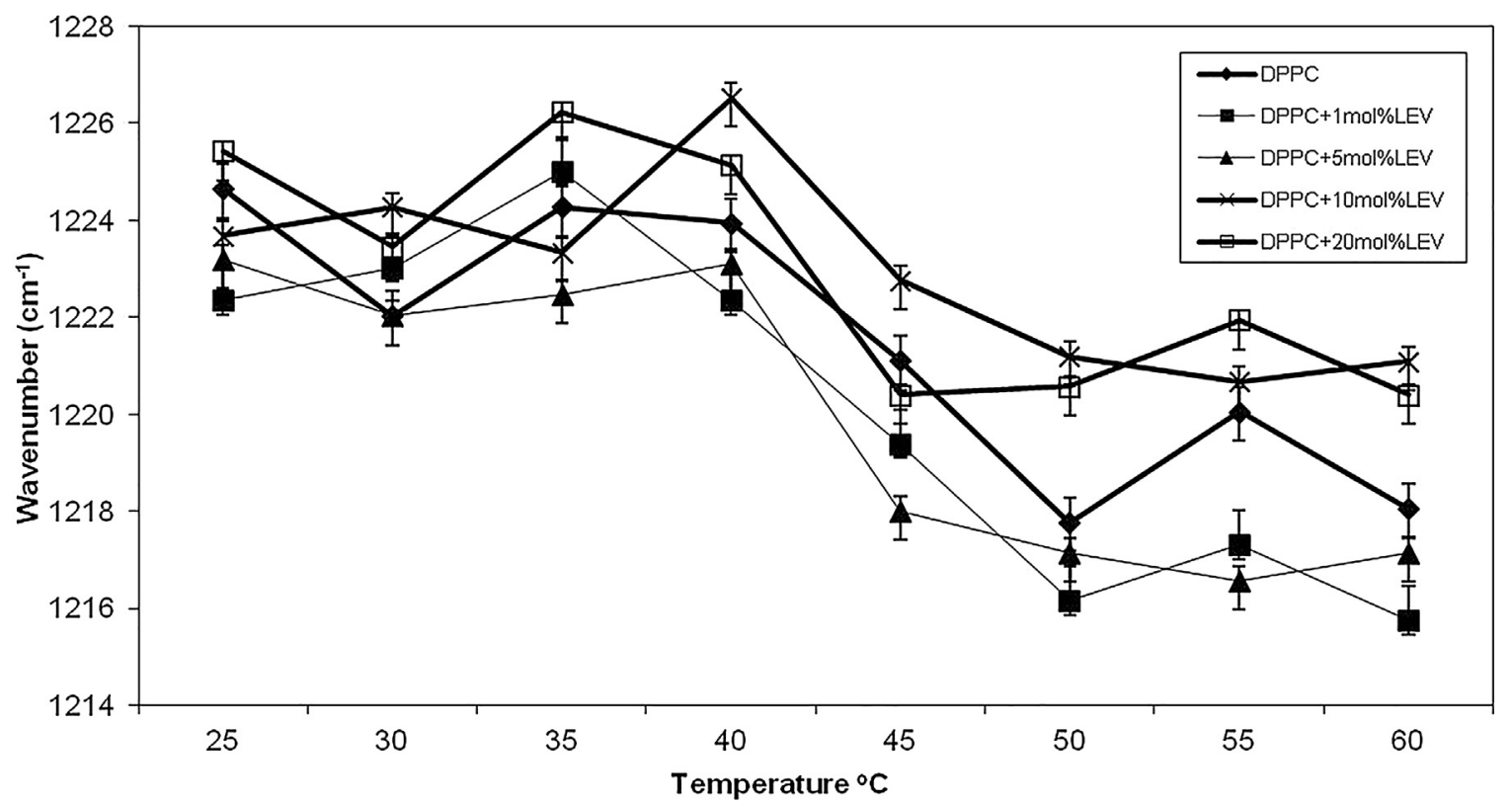

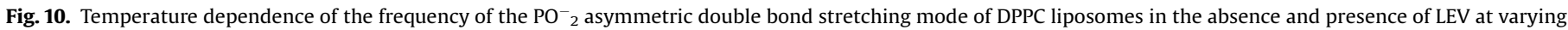
concentrations.

normal phase transition of DPPC. In other words, an increasing proportion of lipid molecules is removed from undergoing gel to fluid phase transition. ${ }^{29}$ This may provide a preferential localization of GBP and LEV in outer hydrophobic cooperative zone of the bilayer, i.e. the region of $C_{1}-C_{8}$ atoms of the acyl chains. Such localization can be achieved by disturbing van der Waals interactions between acyl chains, and thereby a loss of bilayer packing. Furthermore, the decrease in Tm may be also resulted from adsorption of water molecules, and thus reduces the strength of the interactions of adjacent lipid molecules in the bilayer, primarily by the disruption of polar head group-head group interactions with the presence of GBP and LEV. ${ }^{18,20,28,30}$

Interestingly, LEV was found to display concentration dependent behavior on Tm value of DPPC in particular, which may be due to biphasic phase behavior of this agent that refers to two independent interactions within different concentration ranges. It can be described by an initial decrease in the $T \mathrm{~m}$, but later an increase in the $T \mathrm{~m} .{ }^{31}$ The first interaction takes place below the threshold concentration where LEV at 1-5 mol\% preferentially partitions into the liquid crystalline phase, lowering this value. The secondary interaction above the threshold concentration of LEV at 10-20 mol\% stabilizes the phase. Tm value can also be re-enhanced in comparison to the concentration below threshold as found in the current study. The increase in Tm of DPPC in the presence of 1020 mol\% of LEV may suggest stabilization of bilayer. The oriented insertion of the hydrophobic part of LEV into the bilayer above threshold concentration a consequent increase in the order of the lipidic hydrocarbon chains in the gel state, may be responsible for this behavior. ${ }^{32}$ Such phenomenon can be also explained as being partly due to the shielding of phospholipid head groups from each other resulting in reduced repulsive interaction, ${ }^{33}$ which was also monitored by an increase in the frequency values of $\mathrm{C}=\mathrm{O}$ and $\mathrm{PO}^{-}{ }_{2}$ groups of lipids in FT-IR study. Indeed, in this case, hydrophobic interactions between LEV at $10-20 \mathrm{~mol} \%$ concentrations and lipids act cooperatively to increase the local order and the rigidity of the membrane; as a consequence, $\mathrm{Tm}$ is expected to increase and cooperativity is expected to increased (sharpness of the peak). On the other hand, because of the hydrophobic interactions the lipid molecules surrounding LEV at high concentrations are blocked and are not subject to the transition as the temperature increases. In such a system, the total energy involved in the transition is decreased with respect to the pure DPPC because the number of lipid molecules involved in the temperature-induced transition is reduced. On the other hand, it is worthwhile drawing attention that with increasing concentration of GBP decreased Tm became closer to the value of main transition of pure DPPC MLVs as shown in Table 1. If it is assumed that this trend will be obtained for above $20 \mathrm{~mol} \%$ of GBP, the similar behavior as LEV could be seen in case of GBP. This would also suggest that threshold concentration of GBP showing biphasic effect might be above $20 \mathrm{~mol} \%$.

The character of phase transition parameters alteration induced by presence of increasing amounts of GBP and LEV may suggest that the lipid bilayer undergoes lipid arrangements according to chemical and structural features of GBP and LEV ${ }^{34}$. In order to clarify whether GBP and LEV interact with glycerol and head groups of lipids, the frequency values $\mathrm{C}=\mathrm{O}$ and $\mathrm{PO}^{-}{ }_{2}$ symmetric stretching bands in the scope of FT-IR study were monitored. Hydrogen bond donor and acceptor count of GBP (2 and 3) and LEV ( 1 and 2 ) and their solubility in water $4.5 \mathrm{~g} / \mathrm{L}$ for $\mathrm{GBP}^{35}, 10 \mathrm{~g} / \mathrm{L}$ for $\mathrm{LEV}^{36}$ suggest that they can locate along with carbonyl groups and first $C$ atoms of the acyl chains of PCs, forming hydrogen bonds with carbonyl esters and phosphate groups. ${ }^{37}$ The decreased frequency values of both $\mathrm{C}=\mathrm{O}$ and $\mathrm{PO}^{-}{ }_{2}$ symmetric stretching with the addition of GBP (1-5-10-20 mol\%) and LEV (1-5 mol\%) may imply that the carbonyl groups of the glycerol backbone and head groups of phospholipids are favored hydrogen bonding sites for these drugs. This may also show that new $\mathrm{H}$ bonds are formed. This may introduce difference in the packing of phospholipid molecules. The observation for GBP is in line with X-ray analysis study that had reported that GBP, with its three polymorphs $(\alpha-\beta-\gamma-)$ have hydrogen bonding capacity due to having $\mathrm{N}-\mathrm{H}$ groups. ${ }^{38}$ Furthermore, the works on peptides incorporated with GBP reveal that conformation of GBP is stereochemically constrained in order to adopt folded conformations, resulting in the formation of intramolecular hydrogen bonded structures. ${ }^{39}$ Similar X-ray structural research on co-crystals of LEV has also reported that LEV displays $\mathrm{NH}-\mathrm{O}$ hydrogen bonding. ${ }^{40}$ Hence, the hydrogen bonding can occur in between the $\mathrm{C}=\mathrm{O}$ and $\mathrm{PO}^{-}{ }_{2}$ groups of DPPC and either with $\mathrm{N}-\mathrm{H}$ groups of GBP and LEV or water molecules rather than lipids each other. The increase in hydrogen bonding 
capacity in $\mathrm{C}=\mathrm{O}$ and $\mathrm{PO}^{-}$groups of DPPC lipids may cause an increment in the head group volume, which in turn reduces the order of the hydrocarbon chains ${ }^{41}$, which was obtained from the frequency values of $\mathrm{CH}_{2}$ asymmetric modes in FT-IR study. This occurrence leads to increased chain tilting and creates energetically unfavorable voids in the hydrocarbon region of membranes. ${ }^{42}$ It may also reflect a decrease in phase transition temperature Tm temperature as also found in DSC data for GBP (1-5-10-20 mol\%) and LEV (1-5 mol\%). On the other hand, with the addition LEV at 10-20 mol\% caused a decrease in hydration state of glycerol and head groups of DPPC lipids. This might be resulted from that head groups of lipids might prefertially interact with each other rather than LEV or water molecules, and thereby causing tight packing of hydrophilic part of lipids. This occurrence may reflect an increase in Tm value and lipid order, also showing membrane stabilization. 18,20

Besides disruption of van der Waals interactions between acyl chains, trans/gauche rotameric energy of lipids has also an influence on transition enthalpies values of GBP and LEV. ${ }^{21}$ Monitoring the frequency changes of the $\mathrm{CH}_{2}$ asymmetric stretching mode revealed that GBP at all concentrations and LEV at $1-5 \mathrm{~mol} \%$ concentrations lowered trans and/or increased gauche conformers within the system indicating an disordered state. ${ }^{18,20}$ On the other hand, LEV at $10-20 \mathrm{~mol} \%$ led to an increment in trans conformers, which may mean ordered state of membrane structure. A higher order of acyl chain packing leads to greater stability and increased Tm obtained from DSC data. The stabilization effect of LEV at $10-20 \mathrm{~mol} \%$ on PC lipids may represent to recover and/or to prevent disordered epileptic membrane systems. The interaction of GBP and LEV with hydrophobic core together with changes enthalpy values compared to pure DPPC MLVs can be also related to alterations in fluidity of lipid bilayers. ${ }^{43}$

The studies have demonstrated the changes in lipid fluidity in tissue membranes ${ }^{44-46}$ upon occurrence of epileptic condition. Some reported less fluid, the other stated more fluid membrane systems induced by epileptic seizures. These contradictory studies examined with different membrane systems with varied lipid composition. The stabilizing and destabilizing impacts of GBP and LEV at different concentrations reported in this study may suggest preventive effect of these agents from epileptic seizures in different membrane systems. Additionally, less fluid membrane structure induced by $1-5 \mathrm{~mol} \%$ of LEV obtained in the present study is in line with the reports that have shown the reducing effect of LEV on blood brain barrier permeability. ${ }^{16}$ However, it should be again taken into consideration that the mentioned research were performed on natural membranes systems containing very different kinds of lipids in addition to PCs. More studies should be performed on purified brain cell membranes to further clarify such effects, particularly, bearing concentration dependent effect of LEV in mind.

There are several types of membrane proteins such as membrane-bound proteins play significant role in the generation of excessive nerve cell activity during epileptic seizures. ${ }^{47}$ Peripheral membrane proteins contact with lipid head groups by electrostatic and hydrogen-bond interactions. While, intergral membrane proteins are in interaction with fatty acyl chains of phospholipids through van der Walls and hydrophobic matching. 48 Considering this, all parameters including physical properties and hydrogen state of lipids, lipid order and fluidity may influence structure and function of both peripheral and integral membrane proteins. ${ }^{49}$ The obtained results in the present study showed GBP and LEV at different concentrations induced variations in packing of PC lipids depending on acyl chains, glycerol and head group regions. All alterations in lipid packing may directly affect the interactions of lipids with hydrophobic and hydrophilic residues of proteins. Indeed, the structures adopted by the parts of a membrane protein that are located in the lipid head group region are determined, in part, by hydrogen bonding to the head groups. ${ }^{50}$ The findings about the interaction of GBP and LEV with glycerol backbone and head groups of lipids may indicate that they have the potential to change structure, relatively function, of peripheral proteins. Similarly, the localization of these drugs within hydrophobic core of bilayer may also suggest that they have the potential to change transmembrane segments of integral proteins. Nevertheless, the changes in lipid packing and fluidity induced by GBP and LEV may represent their influence the membrane's transport properties. It should be noted that all the effects of LEV is concentration dependent, evidence from our findings.

\section{Conclusion}

With the aiming to provide knowledge about how GBP and LEV incorporate with membrane lipids, the concentration dependent effects of such drugs on structure and dynamics of DPPC MLVs were investigated in the present study. The results demonstrated that depending on concentrations both GBP and LEV incorporate into DPPC MLVs, perturbing the packing of lipids and affecting their thermotropic properties. The binding affinity of GBP and LEV to acyl chains and hydrophilic parts of lipids was found to highly correlate with lipid-water partition and their solubility degree in water.

All these findings should be also considered as contributing factors to elucidate the physiological action mechanisms of GBP and LEV. However, as a perspective, the effects of GBP and LEV on isolated lipids from brain tissue need to be further investigated for better understanding of their actions on biological membrane systems with various lipid compositions. It would be of great importance in order to get better understanding of GBP and LEVmembrane interactions for efficient and correct usage. On the other hand, the obtained results revealing interaction of GBP and LEV with PC lipids may also offer potential use of these liposomes in order to increase efficacy and bioavailability of such agents. In particular, when considering highly structural integrity of blood brain barrier which may impede the delivery of GBP and LEV to potential target sites in the brain, encapsulation of the drugs at low concentrations ( 1 and $5 \mathrm{~mol} \%$ ) with such lipids may be beneficial. Therefore, the side effects of GBP and LEV given at high concentrations could be prevented. However, in order to practice this approach clinically, effectiveness of encapsulated GBP and LEV should be tested against different types of seizures seen in human epilepsy.

\section{Conflict of interest}

None.

\section{References}

1. Lason W, Chlebicka M, Rejdak K. Research advances in basic mechanisms of seizures and antiepileptic drug action. Pharmacol Rep. 2013;65:787-801.

2. Honarmand A, Safavi M, Zare M. Gabapentin: an update of its pharmacological properties and therapeutic use in epilepsy. J Res Med Sci. 2011;16(8):10621069.

3. Cai K, Nanga R, Lamprou L, et al. The Impact of gabapentin administration on brain GABA and glutamate concentrations: a 7T ${ }^{1} \mathrm{H}-\mathrm{MRS}$ study. Neuropsychopharmacology. 2012;37:2764-2771.

4. Kuzniecky R, Ho S, Pan J, et al. Modulation of cerebral GABA by topiramate, lamotrigine, and gabapentin in healthy adults. Neurology. 2002;58:368-372.

5. Taylor CP, Gee NS, Su T, et al. A summary of mechanistic hypotheses of gabapentin pharmacology. Epilepsy Res. 1998;29:233-249.

6. Davies A, Hendrich J, Van Minh AT, Wratten J, Dolphin DL. Functional biology of the alpha(2)delta subunits of voltage-gated calcium channels. Trends Pharmacol Sci. 2007;28:220-228.

7. Farber NB, Jiang XP, Heinkel C, Nemmers B. Antiepileptic drugs and agents that inhibit voltage-gated sodium channels prevent NMDA antagonist neurotoxicity. Mol Psychiatry. 2002;7:726-733. 
8. Abdel-Wahab B, Shaikh IA, Khateeb MM, Habeeb M. Omega 3 polyunsaturated fatty acids enhance the protective effect of levetiracetam against seizures, cognitive impairment and hippocampal oxidative DNA damage in young kindled rats. Pharmacol Biochem Behav. 2015;135:105-113.

9. Lyseng-Williamson KA. Levetiracetam: a review of its use in epilepsy. Drugs. 2011;71:489-514.

10. Lynch BA, Lambeng N, Nocka K, et al. The synaptic vesicle protein SV2 A is the binding site for the antiepileptic drug levetiracetam. Proc Natl Acad Sci USA. 2004;101:9861-9866.

11. Lee CY, Chen CC, Liou H. Levetiracetam inhibits glutamate transmission through presynaptic P/Q-type calcium channels on the granule cells of the dentate gyrus. Br J Pharmacol. 2009;158:1753-1762.

12. Ribeiro MMB, Melo MN, Serrano I, Santos N, Castanho M. Drug-lipid interaction evaluation: why a 19th century solution? Trends Pharmacol Sci. 2010;31:449-454.

13. Peetla C, Stine A, Labhasetwar V. Biophysical interactions with model lipid membranes: applications in drug discovery and drug delivery. Mol Pharm. 2009;6:1264-1276.

14. Shrestha H, Bala R, Arora S. Lipid-based drug delivery systems. J Pharm. 2014;801820:1-10

15. Luer MS, Hamani C, Dujovny MD, et al. Saturable transport of gabapentin at the blood-brain barrier. Neurol Res. 1999;21:559-562.

16. Gurses C, Ekizoglu O, Orhan N, Kaya M. Levetiracetam decreases the seizure activity and blood-brain barrier permeability in pentylenetetrazole-kindled rats with cortical dysplasia. Brain Res. 2009;1281:71-83.

17. Boltz JR, Feigenson GW. A novel strategy for the preparation of liposomes: rapid solvent exchange. Biochim Biophys Acta. 1999;1417:232-245.

18. Turker S, Wassall S, Stillwell W, Severcan F. Convulsant agent pentylenetetrazol does not alter the structural and dynamical properties of dipalmitoylphosphatidylcholine model membranes. J Pharm Biomed Anal. 2011;54:379-386.

19. Barroso R, Basso G, Costa-Filho A. Interactions of the antimalarial amodiaquine with lipid model membranes. Chem Phys Lipids. 2015;186:68-78.

20. Ergun S, Demir P, Uzbay T, Severcan F. Agomelatine strongly interacts with zwitterionic DPPC and charged DPPG membranes. Biochim Biophys Acta. 2014; 1838:2798-2806.

21. Potamitis C, Chatzigeorgiou P, Siapi E, et al. Interactions of the AT1 antagonist valsartan with dipalmitoyl-phosphatidylcholine bilayers. Biochimica et Biophysica Acta-Biomembrane. 2011;1808:1753-1763.

22. Bilge D, Kazancı N, Severcan F. Acyl chain length and charge effect on Tamoxifen-lipid model membrane interactions. J Mol Struct. 2013;1040:7582.

23. Sangster J. LOGKOW Database A. Databank of Evaluated Octanol-water Partition Coefficients P. Log. . [Accessed 29 January 2016] http://logkow.cisti.nrc.ca/ logkow/search.html,.

24. Fay MA, Sheth RD, Gidal E. Oral absorption kinetics of levetricetam: the effect of mixing with food or enteral nutrition formulas. Clin Ther. 2005;2:594-598.

25. Mavromoustakos T, Theodoropoulou E, Yang DP. The use of high-resolution solid-state NMR spectroscopy and differential scanning calorimetry to study interactions of anaesthetic steroids with membrane. Biochim Biophys Acta. 1997; $1328: 65-73$

26. Heimburg T. A model for the lipid pretransition: coupling of ripple formation with the chain-melting transition. Biophys J. 2000;78:1154-1165.

27. Engelke M, Jessel R, Wiechmann A, Diehl H. Effect of inhalation anesthetic on the phase behavior, permeability and order of phosphatidylcholine bilayers. Biophys Chem. 1997;67:127-138.

28. Moore DJ, Sills RH, Mendelsohn R. Peroxidation of erythrocytes: FTIR spectroscopy studies of extracted lipids, isolated membranes, and intact cells. Biospectra J. 1995; 1:133-140.
29. Wiedmann TS, Trouard T, Shekar S, Polikandritou M, Rahman YE. Interaction of cyclosporine A with dipalmitoylphosphatidylcholine. Biochim Biophys Acta. 1990; 1023:12-18.

30. Liu KZ, Schultz CP, Johnston JB, et al. Infrared spectroscopic study of bryostatin I-induced membrane alterations in a B-CLL cell line. Leukemia. 1999;13:12731280.

31. Rowe ES. Lipid Chain length and temperature dependence of ethanolphosphatidylcholine interaction. Biochemical. 1983;22:3299-3305.

32. Grasso D, Milardi D, La Rosaa C, Rizzarellia E. DSC study of the interaction of the prion peptide PrP 106-126 with artificial membranes. New J Chem. 2001:25:1543-1548.

33. Sillerud LO, Schafer DE, Yu RK, Konigsberg WH. Calorimetric properties of mixtures of ganglioside G(M1) and dipalmitoylphosphatidylcholine. J Biol Chem. 1979;254:10876-10880.

34. Jain MK, Wu NM. Effects of small molecules on the dipalmitoyl lecithin liposomal bilayer: III. Phase transitions of lipid bilayers. J Membr Biol. 1977;34:157-201.

35. US EPA. Estimation Program Interface (EPI) Suite. . [Accessed 29 January 2016] http://www. epa.gov/oppt/exposure/pubs/episuitedl.htm.

36. Physicians Desk Reference. 61st ed. Montvale, New Jersey: Thomson PDR; 2007.

37. Lombardi D, Cuenoud B, Kramer SD. Lipid membrane interactions of indacaterol and salmeterol: do they influence their pharmacological properties. Eur J Pharm Sci. 2009;38:533-547.

38. Ibers JA. Gabapentin and gabapentin monohydrate. Acta Crystallogr C. 2001;57:641-643.

39. Vasudev P, Ananda K, Chatterjee S, Aravinda S, Shamala N, Balaram P. Hybrid peptide design Hydrogen bonded conformations in peptides containing the stereochemically constrained gamma-amino acid residue, gabapentin. J Am Chem Soc. 2007:129:4039-4048.

40. Springuel G, Norberg B, Robeyns K, Wouters J, Leyssens T. Advances in pharmaceutical co-crystal screening: effective co-crystal screening through structural resemblance. Cryst Growth Des. 2012;12:475-484.

41. Vierl U, Löbbecke L, Nagel N, Cevc G. Solute effects on the colloidal and phase behavior of lipid bilayer membranes: ethanoldipalmitoylphosphatidylcholine. Mixtures Biophys J. 1994;67:1067.

42. Ahl PL, Perkins WR. Interdigitation-Fusion liposomes. Methods Enzymol. 2003;367:80-98.

43. Brandenburg K, Seydel U. Investigation into the fluidity of lipopolysaccharide and free lipid A membrane systems by Fourier-transform infrared spectroscopy and differential scanning calorimetry. Eur J Biochem. 1990;191:229-236.

44. Turker S, Severcan M, Ilbay G, Severcan F. Epileptic seizures induce structural and functional alterations on brain tissue membranes. Biochimica et Biophysica Acta-Biomembranes. 2014;1838:3088-3096.

45. Patel M. Mitochondrial dysfunction and oxidative stress: cause and consequence of epileptic seizures. Free Radical Biol Med. 2004·37:1951-1962.

46. Acharya MM, Katyare SS. Structural functional alterations in mitochondrial membrane in picrotoxin-induced epileptic rat brain. Exp Neurol. 2005;192:7988.

47. Kessler RC, Berglund P, Demler O, et al. The epidemiology of major depressive disorder: results from the National Comorbidity Survey Replication (NCS-R). JAMA. 2003;289:3095-3105.

48. Pickholz M, Oliveira ON, Skaf MS. Interactions of chlorpromazine with phospholipid monolayers: effects of the ionization state of the drug. Biophys Chem. 2007; 125:425-434.

49. Lu JX, Sharpe S, Ghirlando R, Yau WM, Tycko R. Oligomerization state and supramolecular structure of the HIV-1 Vpu protein transmembrane segment in phospholipid bilayers. Protein Sci. 2010;19:1877-1896.

50. Preetha A, Huilgol N, Banerjee R. Comparison of paclitaxel penetration in normal and cancerous cervical model monolayer membranes. Colloids Surf B. 2006;53:179-186. 